\title{
Ceruloplasmin and superoxide dismutase (SODI) in heterozygotes for Wilson disease: A case control study
}

\author{
Gudlaug Tórsdóttir ${ }^{1,2}$ \\ Grétar Gudmundsson ${ }^{3}$ \\ Jakob Kristinsson' \\ Jón Snaedal ${ }^{2}$ \\ Torkell Jóhannesson' \\ 'Institute of Pharmacy, Pharmacology \\ and Toxicology, Department of \\ Pharmacology and Toxicology, \\ University of Iceland, Reykjavík, \\ Iceland; ${ }^{2}$ Department of Geriatrics; \\ ${ }^{3}$ Department of Neurology, Landspítali \\ - University Hospital, Reykjavík, \\ Iceland
}

\begin{abstract}
At the time of this study, there were five known patients with Wilson disease (WD) in Iceland. The mutation, a 7-bp deletion in exon 7 on chromosome 13 for WD, is only known in Iceland. In twenty healthy Icelandic heterozygotes for WD and their age- and gender-matched controls, copper concentration in plasma, ceruloplasmin (CP) concentration, CP oxidative activity and CP-specific oxidative activity in serum and superoxide dismutase (SOD1) activity in erythrocytes were determined. The same determinations were done on the five WD patients. There was no significant difference in these parameters between the heterozygotes and the controls, although an inclination toward lower CP determinations and higher SOD1 activity in the heterozygotes was noted. As expected the WD patients were low on the copper and CP parameters, but their SOD1 activity was within the upper normal range. In conclusion, the CP parameters and SOD1 activity are within the normal range in Icelandic heterozygotes for WD, although with a trend toward mild dyshomeostasis. This may indicate subclinical copper retention in the heterozygotes, but a bigger study group is needed to confirm this.
\end{abstract}

Keywords: ceruloplasmin, SOD1, heterozygotes, Wilson disease

\section{Introduction}

Wilson disease (WD) is characterized by decreased biliary copper excretion and reduced incorporation of copper in apoceruloplasmin, resulting in lower levels of ceruloplasmin (CP) and copper in serum but copper accumulation in various organs and tissues. WD is an autosomal recessive disease of copper transport, and the gene for WD has been mapped to the short arm of chromosome 13. ${ }^{1}$ It was cloned and coded for as a putative copper transporting P-type ATPase (ATP7B)., ${ }^{2,3}$ WD may present under a variety of clinical symptoms, most commonly from liver and/or as neuropsychiatric disturbances.

The incidence of hypoceruloplasminemia in heterozygotes for WD is not known, but they usually have a normal $\mathrm{CP}$ concentration in serum, although it may be slightly reduced. ${ }^{4,5}$ The heterozygotes are most commonly clinically asymptomatic and have a normal copper concentration in serum. They may however, show some signs of disturbed copper metabolism. While patients with WD do not incorporate radioactive copper $\left({ }^{64} \mathrm{Cu}\right.$ and $\left.{ }^{67} \mathrm{Cu}\right)$ into $\mathrm{CP}$, the heterozygotes incorporate the isotopes into $\mathrm{CP}$ at a rate between that of normal individuals and WD patients. ${ }^{4}$

$\mathrm{Cu}-\mathrm{Zn}$ superoxide dismutase (SOD1) activity in erythrocytes is known to be lowered in copper deficiency, but the correlation with copper and $\mathrm{CP}$ in serum is poor. ${ }^{6}$ Copper-chelating treatment has been found to lower SOD1 activity in erythrocytes, and it was concluded that the SOD1 activity in erythrocytes reflects the availability of intracellular copper. ${ }^{6}$ SOD1 activity was found to be the same in three untreated WD patients, compared with controls, but was lowered after treatment with D-penicillamine. ${ }^{7}$ To our knowledge no study has been done on SOD1 in heterozygotes for WD. 
Gamma glutamyl transpeptidase (GGT) is localised predominantly within the cytoplasm of hepatocytes and biliary epithelial cells of the liver. It has been recognized as a useful marker of liver disease. ${ }^{8}$ As CP in serum is produced in hepatocytes, the determination of GGT activity in serum is of use, as damage of hepatocytes can be a cause for changes in $\mathrm{CP}$ parameters.

At the time of this study, there were five living Wilson patients in Iceland. All of the known patients in Iceland have had the same mutation, a 7-bp deletion in exon 7 on chromosome 13 , and this mutation is not known elsewhere in the world. ${ }^{9}$

This study was undertaken in order to elucidate whether the heterozygotes for the unique Icelandic type of WD had any particular derangement of their copper metabolism.

\section{Material and methods}

The five known Icelandic WD patients and 20 healthy, first-degree relatives of the WD patients (heterozygotes) were traced (parents, siblings, and children of the patients). The mean age of the heterozygotes was 41 years (range 21-70), nine males and 11 females. Each heterozygote had healthy age- and gender-matched control.

The blood samples were taken in the morning in the fasting state, and the blood sampling units used were specially prepared for metal analysis $\left(\right.$ Sarstédt $\left.^{\circledR}\right)$. The same blood sampling procedure was used for the WD patients, heterozygotes and controls. All the WD patients were on copper-chelating treatment.

The study was approved by the Icelandic National Bioethics Committee (01-056-S1) and registered by The Data Protection Authority.

\section{Determination of gamma glutamyl transpeptidase}

GGT activity in serum, expressed as units/1, was determined at the Icelandic University Hospital Laboratories, on Vitros 950, Ortho-Clinical Diagnostics, Rochester, NY, USA. GGT catalyzes the transfer of the gamma-glutamyl portion of L-gamma-glutamyl-p-nitroanilide to glycylglycine, simultaneously producing $\mathrm{p}$-nitroaniline. The rate of change in reflection density is measured and used to calculate the enzyme activity of GGT. The upper limit of GGT in healthy subjects is 70 units $/ 1$ at these laboratories.

\section{Determination of copper in plasma}

A solution of $0.5 \mathrm{ml}$ plasma, $5 \mathrm{ml}$ of double distilled water, $0.2 \mathrm{ml}$ of concentrated hydrochloric acid (Emerck, Darmstadt,
100318 ) and $0.125 \mathrm{ml}$ of concentrated nitric acid (E Merck, 100456) was pipetted into a Teflon-lined microwave acid digestion bomb. The digestion bomb was heated in a $600 \mathrm{~W}$ microwave oven for $3.25 \mathrm{~min}$. After cooling, $10 \mathrm{ml}$ of distilled water were added and samples taken to analysis. A standard serum from Nycomed Pharma AS, Oslo, Norway, was processed in the same way. Copper in the samples ( $\mu \mathrm{mol} / \mathrm{l}$ plasma) was determined at the Icelandic Fisheries Laboratories, Reykjavík, by atomic absorption spectrophotometry.

\section{Determination of CP concentration}

Ceruloplasmin levels in serum $(\mathrm{mg} / \mathrm{l})$ were determined at the Icelandic University Hospital Laboratories with rate nephelometry immunoassay (Beckman Immunochemistry Systems, ICS-2 Beckman Instruments Inc.). The CP antibody was processed goat sera, which were calibrated with an International Federation of Clinical Chemistry human calibrator. The results are represented as $\mu \mathrm{mol} / 1$ in Table 1 .

\section{Determination of CP oxidative}

\section{activity in serum}

Ceruloplasmin oxidative activity in serum (units/ml) was determined at the Department of Pharmacology and Toxicology, University of Iceland, with the manual kinetic assay described by Boyett and colleagues. ${ }^{10}$ The method is based on the oxidative activity of $\mathrm{CP}$ with o-dianisidine as substrate. The substrate reagent was prepared at this laboratory as follows: $250 \mathrm{mg}$ of o-dianisidine were added to $50 \mathrm{ml}$ of distilled water. $1.0 \mathrm{ml}$ Triton X-100 was added and the solution then topped up to $100 \mathrm{ml}$ with distilled water. Just before use $2.0 \mathrm{ml}$ of the o-dianisidine solution was topped up to $10 \mathrm{ml}$ with acetate buffer solution. $0.15 \mathrm{ml}$ of serum was incubated with $3.0 \mathrm{ml}$ of substrate reagent in a cuvette positioned in a spectrophotometer. The change in absorbance at $460 \mathrm{~nm}$ with time was observed. The enzyme activity was reported as units $/ \mathrm{ml}$, based on the molar absorption coefficient of o-dianisidine consumed.

\section{Determination of superoxide dismutase (SODI) activity in erythrocytes}

Superoxide dismutase activity in erythrocytes (SOD525 units) was determined at the Department of Pharmacology and Toxicology, University of Iceland, with a spectrophotometric assay (Bioxytech ${ }^{\circledR}$, SOD-525) as described by Nebot and colleagues. ${ }^{11}$ The superoxide dismutase activity in the erythrocytes of both the patients and their controls was determined the same day. The assay is based on the SOD-mediated increase in the rate of auto-oxidation of 
Table I GGT activity, copper concentration, CP concentration, CP oxidative activity, CP specific oxidative activity and SOD lactivity in 20 heterozygotes for Wilson disease with their age- and gender-matched controls. The number of pairs is indicated and the $P$ values of the comparisons of the means. The null hypothesis was rejected for values $\leq$ than 0.05 . The results of same determinations are also shown in the 5 homozygotes known in Iceland at the time of the study

\begin{tabular}{|c|c|c|c|c|}
\hline Determinations & $\begin{array}{l}\text { Heterozygotes } \\
\text { Mean (range) }\end{array}$ & $\begin{array}{l}\text { Controls } \\
\text { Mean (range) }\end{array}$ & P-values & $\begin{array}{l}\text { Homozygotes } \\
\text { Mean (range) }\end{array}$ \\
\hline GGT units/l (serum) & $25(12-86)$ & $28(16-68)$ & $0.104 * 2$ & $61(14-194)$ \\
\hline Copper conc. $\mu \mathrm{mol} / \mathrm{I}$ (plasma) & $18(11-32)$ & $19(12-33)$ & $0.507^{* * 1}$ & $3.2(2-5)$ \\
\hline CP conc. $\mu \mathrm{mol} / \mathrm{l}$ (serum) & $2.3(1.3-3.6)$ & $2.3(1.4-4.1)$ & 0.5391 & $\alpha$ \\
\hline CP oxidative activity units/ml (serum) & | $37(82-2 \mid 1)$ & $152(65-227)$ & $0.170^{\prime}$ & $29(17-36)$ \\
\hline $\mathrm{CP}$ specific oxidative activity units $/ \mu \mathrm{mol} * 10^{-4}$ (serum) & $6.2(3.7-11.1)$ & $6.9(2.8-9.4)$ & $0.392^{\prime}$ & $\beta$ \\
\hline SODI activity, SOD 525 units (erythrocytes) & $360(265-476)$ & $328(250-437)$ & $0.066 * 2$ & $352(319-376)$ \\
\hline
\end{tabular}

Abbreviations: GGT, gamma glutamyl transpeptidase; CP, ceruloplasmin; SODI, Cu-Zn superoxide dismutase.

Notes: 'Student's two sample t test, in a normally distributed sample; ${ }^{2}$ Wilcoxon's two sample test, were the sample is not normally distributed; *Not normally distributed after logarithmic transformation; **Normally distributed after logarithmic transformation; $\alpha$ Under the det.lim. of the laboratory; $\beta$ Not calculated as the CP concentration is not available.

5,6,6a,11b-tetrahydro-3,9,10-trihydroxybenzofluorene (BXT-01050) in aqueous alkaline solution. The kinetic measurement of 525-nm absorbance is performed for 1 minute upon the addition of BXT-01050. The SOD activity was determined from the ratio of the auto-oxidation measured in the presence and in the absence of the sample. One SOD activity unit (U-525) has been defined as the activity that doubles the auto-oxidation background. The reagents used in the analyses were obtained from Oxis International, Inc., Portland, OR, USA.

\section{Ceruloplasmin specific oxidative activity}

The specific oxidative activity of $\mathrm{CP}$ in serum (oxidative activity relative to mass) was calculated as $\mathrm{CP}$ oxidative activity, units $/ \mathrm{ml}$, divided by the value of $\mathrm{CP}$ concentration. This is represented as units $/ \mu \mathrm{mol} * 10^{-4}$ in Table 1.

\section{Statistics}

Ceruloplasmin concentration, oxidative activity and specific oxidative activity were normally distributed in the controls. The GGT activity and copper concentration did not pass normality tests (D'Augostino and Pearson omnibus and Shapiro-Wilk), but did so after the transformation to logarithmic figures. The SOD1 activity did not pass the normality tests, and transformation of the SOD1 values to logarithmic figures did not change this. For this reason, SOD1 activity in heterozygotes and the age- and gender-matched controls were compared by using Wilcoxon's two-sample test. The student's two-way t-test was used for comparing the results of the logarithmic figures of GGT and copper and for the determinations of $\mathrm{CP}$ concentration, oxidative activity and specific oxidative activity in the heterozygotes and their age- and gender-matched controls.

The calculations were done by GraphPad Prism ${ }^{\circledR}$, but Figure 1 was made by Med $\mathrm{Calc}^{\circledR}$. The null hypothesis was rejected for $\mathrm{P}$ values $\leq 0.05$.

\section{Results}

The results of the determinations on the WD patients, heterozygotes, and their controls are represented in Table 1 and Figure 1.

\section{Discussion}

There was no significant difference in the activity of GGT in the heterozygotes compared with the controls. One WD patient had raised GGT. This patient was diagnosed with liver cancer shortly after the time of the study, probably as a late complication of WD.

All the Icelandic WD patients had lowered copper in plasma and lowered CP concentration and CP activity in serum. The SOD1 activity in erythrocytes was normal in the WD patients, although the SOD1 activity in all but one patient was above the median value of the control group for the heterozygotes (Figure 1). This could reflect mild intracellular copper retention in spite of the lowered copper in plasma, lowered $\mathrm{CP}$ determinations in serum and the copper-chelating treatment in the WD patients.

In this study the $\mathrm{CP}$ concentration, $\mathrm{CP}$ oxidative activity and CP specific oxidative activity (activity related to mass, reflecting the quality of the protein) in serum were not significantly different in the heterozygotes compared with the control group. This is in line with earlier studies. ${ }^{4,5,12}$ Furthermore, the copper in plasma was not significantly 


\section{SOD1 activity in erythrocytes}

SOD-525 units

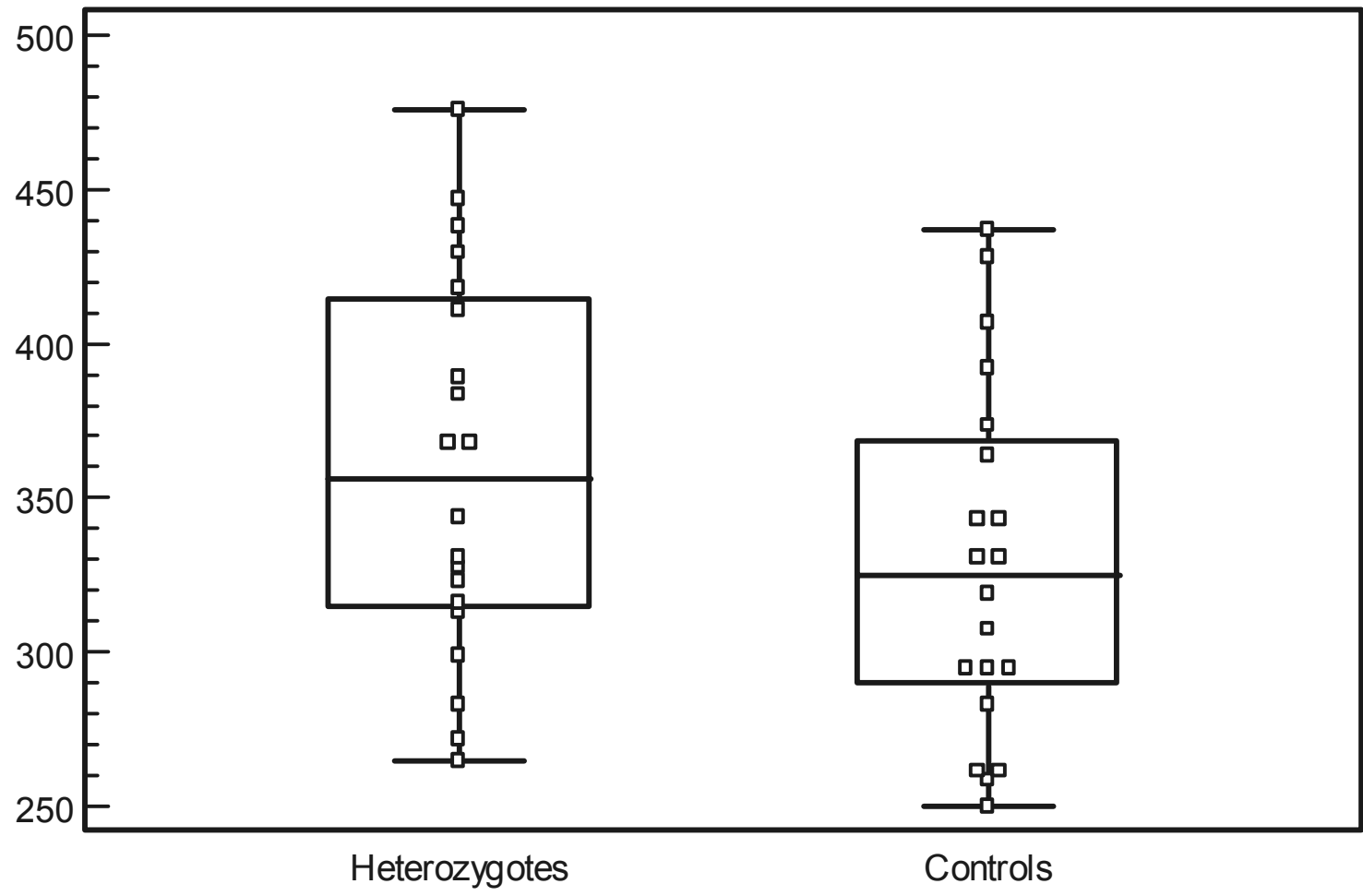

Figure I Distribution of individual values of SODI activity in heterozygotes and controls (SOD-525 units in erythrocytes). The central box represents the values from the 25th to the 75th percentile (314-414 in heterozygotes and 289-368 in the controls). The middle line represents the median in the patients and the controls (354 and 325), respectively. The horizontal lines represent the lowest and highest value, (265 and 476) in heterozygotes, (250 and 437) in controls.

Abbreviation: SODI, Cu-Zn superoxide dismutase.

different between the heterozygotes compared with controls. Still, it was possible to see a trend toward lower CP oxidative activity in the heterozygotes. As described in the Introduction, Cox and colleagues showed that after an oral and intravenous dose of labelled copper $\left({ }^{64} \mathrm{Cu}\right.$ and ${ }^{67} \mathrm{Cu}$ ), the incorporation rates into $\mathrm{CP}$ were slower in heterozygotes than in normal controls but faster than in the homozygotes for WD. ${ }^{4}$ In a study by Gollan and colleagues, lowered CP-specific oxidative activity was encountered in two families with WD, in both the patients (homozygotes) and the heterozygotes, while this was not the case in other WD families studied by these authors. ${ }^{13}$ This reflects that the quality of $\mathrm{CP}$ can vary between different families with WD (reflecting different mutations of the ATP7B). The results of our study (although not significant) point toward lesser quality of the CP in the heterozygotes in the Icelandic families with WD compared with individuals with no family history of WD.

The Icelandic heterozygotes did not show any symptoms in either the liver or the nervous system, indicating that a slightly deranged copper metabolism does not promote disease. Still, there are reports of mild movement disorders in heterozygotes for WD. ${ }^{14-17}$ As CP concentration and oxidative activity have been found to be significantly lower in Alzheimer disease and Parkinson disease, ${ }^{18-20}$ the question arises whether the heterozygotic relatives of the WD patients are more likely to develop these neurodegenerative diseases compared with the general population, as they get older. In that context, the division of the heterozygotes and their controls into age groups would have been of interest, but this was not considered meaningful in this study as the sample was very small. Our earlier studies have not shown a correlation between the CP concentration or activity in serum or SOD1 activity in erythrocytes and age in healthy individuals, ${ }^{19,20}$ which is in accordance with other authors. ${ }^{21,22}$

SOD1 activity in erythrocytes was not significantly different between the heterozygotes and the controls $(\mathrm{P}=0.066)$, but there was a trend toward higher SOD1 activity in the heterozygotes (Figure 1). The trend toward higher SOD1 activity in the heterozygotes in the present study can reflect a slight tendency to lower copper excretion and thus higher intracellular copper in the heterozygotes compared with controls. 
Thus, taken together, although the Icelandic heterozygotic carriers do not develop WD, they may exhibit mild abnormalities in copper metabolism, as described in the results above. An important limitation is the low power of this study as the sample is small and it would clearly be of interest to investigate bigger groups of heterozygotes for WD to further confirm this trend. Moreover, the heterozygotes in this study are rather young or middle-aged. In a bigger study group it would be relevant to see if neurodegenerative diseases would be more prevalent with rising age in this group than in the general population. The WD patients show similar defects in CP determinations as described earlier from different WD families although the Icelandic mutation of the Wilson gene is unique.

\section{Acknowledgments}

This work was supported by grants from The Memorial Foundation of Helga Jónsdóttir and Sigurlidi Kristjánsson (Medical Division). Our sincerest thanks are also due to Physician-in-Chief Jón Jóhannes Jónsson, PhD, and Elín Ólafsdóttir, MD, at the University Hospital Laboratories for the services rendered there. Furthermore, sincere thanks go to Professor Magnús Jóhannsson, $\mathrm{MD}$, and $\mathrm{PhD}$, at the Institute of Pharmacy, Pharmacology and Toxicology, Department of Pharmacology and Toxicology at the University of Iceland, for assistance with the statistical analysis and to Steinunn Björg Sigurjónsdóttir, biomedical scientist, for her assistance with the analytical procedure. The authors report no conflicts of interest in this work.

\section{References}

1. Ferenci P. Pathophysiology and clinical features of Wilson's disease. Metab Brain Dis. 2004;19:229-239.

2. Bull CP, Gordon TR, Rommens JM, Forbes JR, Cox DW. The Wilson disease gene is a putative copper transporting P-type ATPase similar to the Menkes gene. Nat Genet. 1993;5:327-337.

3. Tanzi RE, Petrukhin K, Chernov I, et al. The Wilson disease gene is a copper transporting ATPase with homology to the Menkes disease gene. Nat Genet. 1993;5:344-350.

4. Cox DW, Clarke Fraser F, Sass-Kortsak A. A genetic study of Wilson's disease: Evidence for heterogeneity. Amer J Hum Genet. 1972;24:646-666.
5. Asonuma $\mathrm{K}$, InomataY, Kasahara $\mathrm{M}$, et al. Living related liver transplantation from heterozygote genetic carriers to children with Wilson's disease. Pediatr Transplant. 1999;3:201-205.

6. Marklund SL, Ek B, Steen L. Effect of long-term penicillinamine therapy on erythrocyte CuZn superoxide dismutase activity. Scand J Clin Lab Invest. 1984;44:13-17.

7. Alexander NM, Benson GD. Superoxide dismutase activity (erythrocuprein) in Wilson's disease. Life Sci. 1975;16:1025-1032.

8. Giannini EG, Testa R, Savarino V. Liver enzyme alteration: a guide for clinicians. CMAJ. 2005;172:367-379.

9. Thomas GR, Jensson Ó, Gudmundsson G, Thorsteinsson L, Cox DW. Wilson's disease in Iceland: A clinical and genetic study. Am J Hum Genet. 1995;56:1140-1146.

10. Boyett JD, Lehmann HP, Beeler MF. Automated assay of ceruloplasmin by kinetic analysis of o-dianisidine oxidation. Acta Clin Chem. 1976;69:233-241.

11. Nebot C, Moutet P, Huet P, Xu JZ, Yadan JC, Chaudiere J. Spectrophotometric assay of superoxide dismutase activity based on the activated autoxidation of tetracyclic cathechol. Anal Biochem. 1993;214:442-451.

12. Gibbs K, Walshe JM. A study of the Caeruloplasmin concentration found in 75 patients with Wilson's disease, their kinship and various control groups. Quart J Med. 1979;191:447-463.

13. Gollan JL, Stocks J, Dormandy TL, Sherlock S. Reduced oxidase activity in the ceruloplasmin of two families with Wilson's disease. J Clin Path. 1977;30:81-83.

14. Quinn NP, Marsden CD. Coincidence of Wilson's disease with other movement disorders in the same family. J Neurol Neurosurg Psychiatry. 1986;49:221-222.

15. Barbosa ER, Kostow UP, Hirsch R, Cancado ER, Scaff M, Canelas HM. Association of Wilson's disease with non-Wilsonian extrapyramidal syndrome in the same family (abstract). Arq Neuropsiquiatr. 1990;48:501-504. (Article in Portuguese).

16. Nicholl DJ, Ferenci P, Polli C, Burdon MB, Pall HS. Wilson disease presenting in a family with an apparent dominant history of tremor. J Neurol Neurosurg Psychiatry. 2001;70:514-516.

17. Kuhn J, Miyajima H, Takahashi Y, et al. Extrapyramidal and cerebellar movement disorder in association with heterozygous ceruloplasmin gene mutation. J Neurol. 2005;252:111-113.

18. Snaedal J, Kristinsson J, Gunnarsdóttir S, Ólafsdóttir Á, Baldvinsson M, Jóhannesson T. Copper, ceruloplasmin and superoxide dismutase in patients with Alzheimer's disease. Dementia. 1998;9:239-242.

19. Tórsdóttir G, Kristinsson J, Sveinbjörnsdóttir S, Snaedal J, Jóhannesson T. Copper, ceruloplasmin, superoxide dismutase and iron parameters in Parkinson's disease. Pharmacol Toxicol. 1999;85:239-243.

20. Tórsdóttir G, Kristinsson J, Sveinbjörnsdóttir S, Snaedal J, Jóhannesson T. Ceruloplasmin and superoxide dismutase (SOD1) in Parkinson's disease: A follow up study. J Neurol Sci. 2006;241:53-58.

21. Kasapoglu M, Ozben T. Alterations of antioxidant enzymes and oxidative stress markers in aging. Exp Gerontol. 2001;36:209-220.

22. Musci G, Bonaccorsi di Patti MC, Fagiolo U, Calabrese L. Age-related changes in human ceruloplasmin. Evidence for oxidative modifications. J Biol Chem. 1993;268:13388-13395. 
